\title{
How the fear of COVID-19 changed the way we look at human faces
}

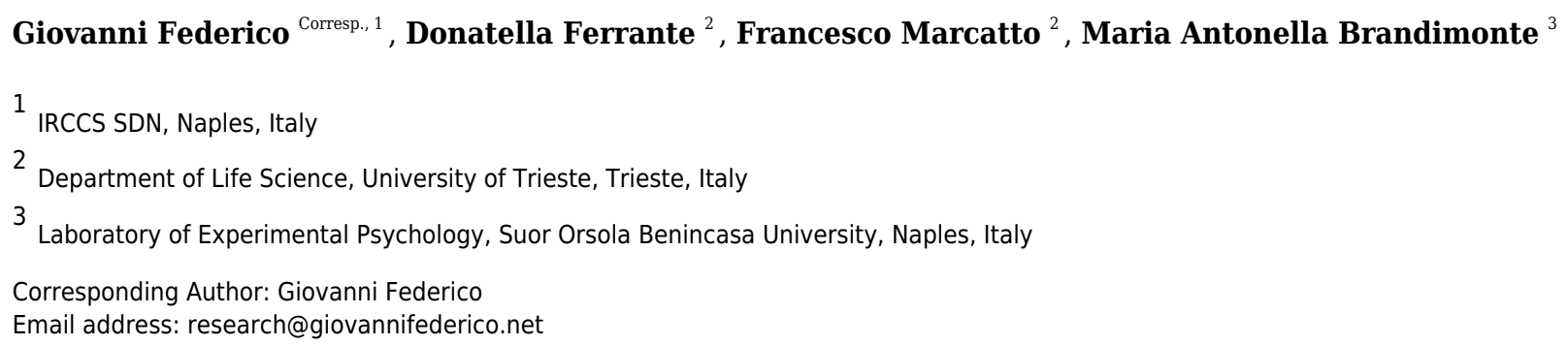

Do we look at persons currently or previously affected by COVID-19 the same way as we do with healthy ones? In this eye-tracking study, we investigated how participants $(N=54)$ looked at faces of individuals presented as "COVID-19 Free", "Sick with COVID-19", or "Recovered from COVID-19". Results showed that participants tend to look at the eyes of COVID-19-free faces longer than at those of both COVID-19-related faces. Crucially, we also found an increase of visual attention for the mouth of the COVID-19-related faces, possibly due to the threatening characterisation of such area as a transmission vehicle for SARS-CoV-2. Thus, by detailing how people dynamically changed the way of looking at faces as a function of the perceived risk of contagion, we provide the first evidence in the literature about the impact of the pandemic on the most basic level of social interaction. 
1 How the fear of COVID-19 changed the way we look at human faces.

2 Giovanni Federico ${ }^{1 *}$, Donatella Ferrante ${ }^{2}$, Francesco Marcatto ${ }^{2}$, Maria Antonella

3 Brandimonte ${ }^{3}$

4

51 IRCCS SDN, Naples, Italy.

62 Department of Life Science, University of Trieste, Italy.

$7{ }^{3}$ Laboratory of Experimental Psychology, Suor Orsola Benincasa University, Naples, 8 Italy.

\section{Abstract}

Do we look at persons currently or previously affected by COVID-19 the same way as we do with healthy ones? In this eye-tracking study, we investigated how participants ( $\mathrm{N}$ = 54) looked at faces of individuals presented as "COVID-19 Free", "Sick with COVID$19 "$, or "Recovered from COVID-19". Results showed that participants tend to look at the eyes of COVID-19-free faces longer than at those of both COVID-19-related faces. Crucially, we also found an increase of visual attention for the mouth of the COVID-19related faces, possibly due to the threatening characterisation of such area as a transmission vehicle for SARS-CoV-2. Thus, by detailing how people dynamically changed the way of looking at faces as a function of the perceived risk of contagion, we provide the first evidence in the literature about the impact of the pandemic on the most basic level of social interaction.

\section{" Correspondence concerning this article should be addressed to:}

Giovanni Federico

IRCCS SDN, Naples, Italy

E-mail: research@giovannifederico.net 


\section{Introduction}

The COVID-19 pandemic caused by SARS-CoV-2 is a global health concern that may cause severe threats to individuals' lives (World Health Organization, 2020a). Alongside the potentially fatal disease and the wide variability of physical health problems caused by the new beta-coronavirus, it is increasingly evident that the pandemic produced strong social, economic, and psychological effects (Bavel et al., 2020; Brooks et al., 2020; Fiorillo \& Gorwood, 2020; Pfefferbaum \& North, 2020). Therefore, in a fast and sadly predictable way, many clinical and psycho/sociological findings related to COVID19's collateral effects have been accumulated. However, much less has been said about how the aftereffects of the current situation may reverberate on non-pathological, daily-life cognitive functioning. After all, if COVID-19 changed the world as we have been knowing it, one might wonder whether and, if so, how the "cognitive interface" between humans and the (post-COVID-19) world may transform.

When thinking about which "sensors" might primarily constitute such a kind of human interface with the world, the response is straightforward: Eyes. Indeed, studying how humans look at their surroundings may provide very useful insights about the cognitive processes underlying a wide variety of human behaviours as well as how people interact with the environment (Federico \& Brandimonte, 2020; Hayhoe \& Ballard, 2005; Henderson, 2017; Liversedge \& Findlay, 2000; Milner \& Goodale, 2006; Rahal \& Fiedler, 2019; Rayner, 1998). From the heterogeneous sets of stimuli present in the environment, there are some that mainly attract the attention of human beings when it comes to interacting with their own kind: others' human faces and, specifically, others' eyes (Senju \& Johnson, 2009a). Indeed, more than nose and mouth, eyes constitute a major visual-attentional target for adults involved in face exploration (Hernandez et al., 2009; Mertens et al., 1993; Walker-Smith et al., 1977). Also, humans tend to shift attention according to others' eye-gaze direction. Thus, as well as capturing and holding attention, eye-gaze may also elicit spatial orienting (for a comprehensive review, see Dalmaso, Gastelli \& Galfano, 2020).

Whereas in many species direct-gaze perception may produce an aversive response (Emery, 2000), the so-called "eye-contact effect" - by describing a broad set 
52 of neurocognitive effects associated with making eye contact with others - seems to be

53 a foundation of human social interaction, hence constituting the basis of social cognition 54 (Csibra \& Gergely, 2006; Kleinke, 1986; Senju et al., 2006; Senju \& Johnson, 2009a). In 55 addition, in the context of self-referential processing (i.e., the way humans process 56 stimuli concerning themselves), direct-gaze perception appears to produce a wide range of social-related effects. Indeed, the so-called "watching-eyes effects" may favour George \& Hietanen, 2016).

Although at different degrees of consciousness, eye-contact effects seem to be governed by the so-called "social brain", an extensive and composite brain network involved in human social interaction (Adolphs, 2009). To date, an atypical eye-contact pattern is one of the most significant symptoms of Autism Spectrum Disorder, which is a disorder that severely affects social functioning (American Psychiatric Association, 2013; Senju \& Johnson, 2009b). Also, the intranasal administration of oxytocin, a neuropeptide mainly implicated in social-communicative function (Insel, 2010), appears to enhance eye contact in both autistic and neurotypical individuals (Auyeung et al., 2015). Thus, the mutual eye-contact experience we do daily when interacting with people probably substantiates one of the most powerful mechanisms to engage others (Senju \& Johnson, 2009b). It seems, therefore, plausible that such a characteristic social behaviour might be affected by how severely COVID-19 impacted our psychosocial functioning, habits, and social interaction (Bagcchi, 2020; Bavel et al., 2020).

As stated above, most people fear the COVID-19 pandemic (Pakpour \& Griffiths, 2020). Fear is a foundation emotion whose function is to protect animals against dangerous, threatening and aversive situations (Misslin, 2003). The neural counterparts of such an emotion are the cortical and subcortical areas implicated in the social brain network (LeDoux, 2003). Indeed, fear's brain networks are in charge of distinct defensive responses (e.g., flight, fight, freezing, avoidance) and may be triggered automatically either by unconditioned or conditioned stimuli (Misslin, 2003). In particular, the activity of the amygdala, an important crossroad of human emotional life, plays a significant role in reading social signals from the face, particularly in modulating direct 
gaze on others (Adolphs, 2010; Hietanen, 2018; Johnson et al., 2005; Kawashima et al., 1999). This is true even for cortically blind patients (Burra et al., 2013). Thus, whereas others' direct gaze typically signal attention and social inclusion (Wirth et al., 2010), threatening stimuli, such as threat-priming faces, should be hardly considered as "social-engaging cues" so that one would likely avoid direct gaze with them (Skuse, 2003). In particular, if COVID-19-infected people were recognised as a possible threat to individual health, one might perceive them as minacious stimuli and, therefore, avoid or reduce direct eye-contact with them. In other words, others' human faces visual exploration might subtly change as an effect of the perceived risk of COVID-19 contagion.

Whereas making a specific experimental hypothesis about the lower duration of eye contact for COVID-19-related faces appears intriguing in itself, it may open the avenue to a further research question: how are other aspects of COVID-19-related faces looked at? Some useful insights might come by considering how threatening stimuli can automatically capture attention, even unconsciously (Lin et al., 2009). Take, for example, the well-known "weapon focus" effect. The higher concentration of a crime eyewitness' attention on the weapon (i.e., the threatening stimulus) may result in a lower ability to remember other crime details (Loftus et al., 1987). Thus, one may ask what kind of threat-related information can be "extracted" from COVID-19-related faces?

According to the World Health Organization, infected people may spread the SARS-CoV-2 by emitting small liquid particles (i.e., larger respiratory "droplets" or smaller "aerosols") through their mouth (World Health Organization, 2020b). Such a piece of critical information - which substantiates the effectiveness of face masks in preventing transmission of SARS-CoV-2 (Bhardwaj \& Agrawal, 2020) - has been repeatedly reported by media, hence becoming part of the semantic knowledge about the post-COVID world. In a sense, the mouth seems to be an important transmission vehicle for the virus, so that one may reasonably predict that this area of a human face might represent a "threatening stimulus" for an interlocutor who, for some reasons, finds himself interacting with a person infected with COVID-19. Thus, whereas, on the one hand, one might expect lower eye contact for COVID-19-related faces, on the other 
113 hand, one may also predict an increase of attention for those face areas that are

114 typically associated with the risk of virus transmission (i.e., the mouth).

115 To test the above hypotheses, in the present study, we analysed by eye-tracking 116 the visual-attentional patterns of participants engaged in an online, ecological, free117 observation task in which they were simply required to look at human-face stimuli 118 generated by Artificial Intelligence. We manipulated the perceived risk of infection 119 prompted by the faces by randomly indicating the immunological status of each face as 120 "COVID-19 free" (i.e., individuals who never contracted the virus), "Sick with COVID-19" 121 (i.e., individuals who are currently infected with COVID-19), or "Recovered from COVID122 19" (i.e., individuals who got COVID-19 but who have now fully recovered). We included 123 the "Recovered from COVID-19" condition to assess whether the COVID-19-related 124 stigma (Bagcchi, 2020) may reverberate on the way people look at faces of patients who survived COVID-19. We thereby conjectured that participants should tend to avoid eye contact for individuals presented as suffering from COVID-19. Also, COVID-19-

127 related faces should attract participants' visuospatial attention towards the areas of the stimulus implicitly recognised as threatening, that is, the mouth. Finally, we included adhoc self-report psychometric measures (i.e., a post-experimental interview; Supplementary Material 1) to assess participants' perceived risk of contagion in relation to their attitude towards the COVID-19 pandemic.

\section{Materials \& Methods}

133

Due to the pandemic situation at the study's date (November 2020), we devised a webbased, online experiment by using custom software and scripts. All participants were safe at home whilst participating in the study and used their own devices (i.e., personal computers or notebooks) to perform the experiment. All experimental procedures followed the ethical standards laid down in the Declaration of Helsinki (1964). Accordingly, the study received approval (approval number: CVD-19-ET) from the Ethics Committee of Suor Orsola Benincasa University (Naples, Italy).

\subsection{Participants}


141 Fifty-four participants (31 females; mean age $=26.46$ years, $S D=5.82$ ) with self-

142 reported normal or corrected-to-normal vision were enrolled in the experiment. 143 Participants were all Caucasians. We calculated the sample size on the basis of 144 previous similar studies (Hernandez et al., 2009; Mertens et al., 1993; Walker-Smith et 145 al., 1977) and by considering an a-priori power analysis (Cohen, 2013; Faul et al., 2007) 146 to detect a small effect size $\left(\eta_{p}^{2}=.20\right)$ within a repeated-measures ANOVA, with a 147 power of .90 and an alpha level of .05 (computed $N=53$ ). All participants had no history 148 of neurological or psychiatric disorders and gave informed consent on their participation 149 by indicating their explicit consent via a specific online form. Four female participants 150 were excluded from data analyses due to their performance above 3.0 SD (outliers).

\subsection{Materials}

152 In this study, we used images of human faces generated by Artificial Intelligence and an 153 ad-hoc post-experimental interview.

154

155

156

157

158

159

160

161

162

163

164

165

166

167 168

\subsubsection{Human faces}

For the experiment, we used 18 images of faces (9 females) generated through machine learning by implementing a generative adversarial network (Karras et al., 2020). By adopting GAN-generated faces, we kept constant the aspect ratio between eyes and mouth, hence maintaining invariant the spatial disposition and size of all anatomical features of the faces. Once images were generated, we erased each one's background by using a proprietary algorithm freely available online at the URL "https://www.remove.bg" (Kaleido Al GmbH, Austria). Then, we grayscaled all the images using the specific function of the KRITA open-source raster graphics editor (v.4.4.1 for Apple macOS). As a result, we obtained 18 images of monochrome faces that appear as real to the human eye. Before enrolling participants and starting with the study, we assessed the degree of authenticity of all the GAN-generated faces by asking independent testers ( $N=10 ; 5$ females) to check whether each face appeared real. All the independent testers considered all the stimuli as real faces. An example of faces involved in the study can be seen in Figure 1. We randomly divided the faces into three 
169 groups that correspond to the three experimental conditions of the experiment: $6 \times$ 170 "COVID-19 Free", 6 x "Sick with COVID-19", 6 x "Recovered from COVID-19". Each 171 experimental condition was matched for face sex (3 females for each condition). We 172 changed the experimental-condition assignment for each group of faces at every 18 173 participants. Thus, at the end of the study $(\mathrm{N}=54)$, each face was displayed in all the 174 experimental conditions, hence controlling for possible effects generated by the specific 175 salience of individual stimuli.

Figure 1 about here

\subsubsection{Post-experimental interview}

178

179

We developed an ad-hoc post-experimental interview (PEI) for the study (Supplementary Material 1). The PEI was introduced to assess participants' risk perception of coming into contact with a COVID-19 patient as well as their propensity to engage in protective behaviours. The risk perception items included six risk judgments adapted from prior research on the psychometric paradigm (Slovic, 1987, 2016), which evaluated the following facets of perceived risk: probability, fear, knowledge, control, and severity. Regarding protective behaviours, participants were asked to evaluate how often they engaged in COVID-19-protective behaviours (such as using the hand sanitiser) in the previous two weeks (6 items) and how often they plan to engage in COVID-19-protective behaviours in the next two weeks (6 items). The PEI also included demographic information (7 items) and questions about prior exposure to COVID-19 (4 items). The PEI was edited and published online by using the Google Forms platform.

\subsection{Procedure}

Each participant received a link through which she could access the online platform for the experiment. Once accessed and prior to the test, participants digitally signed informed consent. Instructions about the experiment and the required experimental setting appeared on the screen. Participants were asked to configure their webcam by following the instruction they read on screen. Then, they completed an eye-tracking calibration procedure by looking at and then clicking on red dots that sequentially 
197 appeared on 40 parts of the screen. Afterwards, the eye-tracking study started. The 198 experimental instructions were: "Now you will see some people's faces. Before each face, you will read if the person is currently SICK WITH COVID, RECOVERED FROM COVID, or NEVER SICK WITH COVID. Please, look at faces in the most natural way possible". Then, the eye-tracking experiment started. A single trial of 6 images of faces related to each experimental condition was administered. Thus, 18 faces were randomly presented according to the experimental visual flow (Figure 2): before each stimulus, a fixation point (i.e., a screen-centred white cross over grey background) was shown for $500 \mathrm{~ms}$. Then, a label indicating the state of health of the coming face appeared for $2000 \mathrm{~ms}$, followed by a second fixation point $(500 \mathrm{~ms})$. Then, a face appeared for $5000 \mathrm{~ms}$. After the face, a black screen appeared for $4000 \mathrm{~ms}$ in order to permit the retina to relax. Each single presentation lasted 12 seconds. Overall, the stimuli presentation lasted 216 seconds. At the end of the stimulation, participants were redirected to a Google Forms webpage to conclude the experiment by responding to the Post Experimental Interview (PEI). At the end of the PEI, participants were asked to evaluate the authenticity of the face stimuli they had previously seen. Globally, the study lasted about 20 minutes for each participant. At the end of the experiment, participants were debriefed regarding the study's purposes and the methods by redirecting them to a specific webpage. All participants reported all stimuli as being real human faces.

\subsection{Apparatus and software paradigms}

Participants used their devices to access the online experiment. Technical prerequisites for participating in the study were using Google Chrome browser and having a personal computer or a notebook with a webcam. We developed the online experiment by using different classes of technologies. For the user-interaction interface (e.g., experimental instructions page, page transitions, etc.), we developed webpages using PHP programming language, JavaScript script language, and MySQL database. Those pages acted as a bridge between the different parts of the experiment, hence guiding participants from the first (i.e., eye-tracking part), to the second (i.e., post-experimental 
226 interview), and then, to the third part of the study (i.e., participants' final evaluation of

227 the stimuli). To acquire participants' gaze data, we used the RealEye.io (RealEye sp. z 228 o.o., Poland) platform, an online eye-tracking technology based on WebGazer library 229 (Papoutsaki et al., 2016). The eye-tracking technology we used (Papoutsaki et al., 230 2016) has been compared with other commercial-grade, high-level eye-tracking 231 systems and sensors (Semmelmann \& Weigelt, 2018). When analyses do not require a 232 very detailed spatial resolution, as in the detail level required by this study, results 233 between systems appear to be comparable. Thus, online webcam-based eye tracking 234 has been proved to be a reliable solution in such a kind of cognitive studies 235 (Semmelmann \& Weigelt, 2018). We included in the study only participants who had 236 devices and webcams capable of obtaining at least a sampling rate of $20 \mathrm{~Hz}$. To 237 construct and publish the post-experimental interview, we used the Google Forms 238 platform. To extract and analyse participants' gaze data, we engineered and developed

239

240

241

242

243

244

245

246

247

248

249

250

251

252

253

254

255 different ad-hoc, custom-made scripts using PHP programming language and the MySQL Database Management System. All the face stimuli involved in the experiment were presented at the best-fitted resolution for participants' displays (auto-resizing stimuli).

\subsection{Gaze-behavioural data}

We analysed gaze-behavioural data in terms of dwell time, that is, the amount of time (expressed in milliseconds) that participants spent looking at different Areas of Interest (AOIs). We thereby defined two distinct AOls: the eye area (i.e., a rectangular area comprising both the eyes) and the mouth area (i.e., a rectangular area including the mouth). For all the stimuli, we maintained fixed both the size and the spatial position of the AOIs. An example of the AOls used in the study can be seen in Figure 3. For the eye-tracking data analyses, we used a two-time-window approach (Federico \& Brandimonte, 2019) aimed at studying the initial (i.e., the first $500 \mathrm{~ms}$ ) and the full visual exploration of the faces (i.e., all the $5000 \mathrm{~ms}$ ). We chose a two-time approach to characterise the time course of participants' visual-spatial exploration. Therefore, we analysed the first-500ms time window to explore the initial stage of participants' visual exploration. Such a time interval acted as an at-a-first-glance indication in data analysis, 
256 highlighting participants' initial fixation patterns as soon as the stimuli appeared.

257 Secondly, we extended the time window of analysis by including participants' full visual 258 exploration (i.e., $5000 \mathrm{~ms}$ ), thus taking into account the effect of time on the

259

260

261

262

263

264

265

266

267

268

269

270

271

272

273

274

275

276

277

278

279

280

281

282

283

284

\section{Figure 3 and 4 about here}

\subsection{Data analyses}

We implemented multiple data analyses. First, we considered how participants looked at the faces as the information we manipulated about their immunological status changed. Thus, we performed two $3 \times 2$ repeated measure analysis of variance with "Immunological Status" ("COVID-19-free" vs. "Sick with COVID-19" vs. "Recovered from COVID-19") as a 3-level factor and "AOIs" (eyes vs. mouth) as a 2-level factor on face fixation duration (expressed in milliseconds) for each time window of analysis (500ms and $5000 \mathrm{~ms}$ ). Secondly, we analysed the post-experimental interviews (PEI) data to explore whether and, if so, to what extent participants' risk perception towards COVID19 and COVID-19-related behaviours and intentions were associated with their gazebehavioural data. Risk perception is known to be a multi-dimensional construct (Slovic, 1987); therefore, we conducted a principal component analysis with varimax rotation to investigate its dimensional structure. Data were previously checked for sphericity and sampling adequacy using the Kaiser-Meyer-Olkin (KMO) and Bartlett tests. The other constructs involved in the PEI were expected to be mono-dimensional, hence we aggregated the responses to obtain, for each participant, a COVID-19-related behaviour score and a COVID-19-related intention score. Pearson correlations were then calculated between the PEI scales and the face fixation duration in each condition, both at $500 \mathrm{~ms}$ and $5000 \mathrm{~ms}$. We used an alpha level of 0.05 for all the analyses and Tukey HSD correction for the post-hoc comparisons.

\section{Results}




\subsection{Eye-tracking results}

286 Eye-tracking data related to participants' initial visual exploration (i.e., 500ms) are 287 summarised in Table 1, whereas data related to the full exploration (i.e., 5000ms) are reported in Table 2.

\subsubsection{Initial visual exploration (first 500ms)}

290

291

292

293

294

295

296

297

298

299

300

301

302

303

304

305

306

307

308

309

310

Regarding the way participants initially visually explored the faces (i.e., within the first $500 \mathrm{~ms}$ ), the results of a repeated-measure analysis of variance showed an interaction between "Immunological Status" and "AOIs", $F(2,98)=3.01, p=0.023, \eta^{2} p=0.074$. Post-hoc pairwise comparisons revealed that eyes of COVID-19-free faces were fixated longer than eyes of COVID-19-related (i.e., "Sick with COVID" and "Recovered from COVID") faces $(p<0.05)$. The interaction effect is shown in Figure $5(A)$. No main effects of "Immunological Status" or "AOIs" were found.

\section{Figure 5 about here}

\subsubsection{Full visual exploration (5000ms)}

By extending the time window of eye-tracking analysis to $5000 \mathrm{~ms}$ to examine participants' full visual exploration, the results of a repeated-measure analysis of variance revealed a main effect of "AOls" on face visual exploration, $F(1,49)=4.19, p=$ $0.046, n^{2} p=0.079$. The main effect was due to longer fixations to Eye AOls than Mouth AOls. However, and most important, an interaction between "Immunological Status" and "AOls" was also found, $F(2,98), p<.001, \eta^{2} p=0.196$. Post-hoc pairwise comparisons revealed that eyes of COVID-19-free faces were fixated longer than eyes of sick-withCOVID-19 faces $(p=0.013)$ and recovered-from-COVID-19 faces $(p<.01)$. Also, the mouth was fixated longer for both sick-with-COVID-19 $(p=0.032)$ and recovered-fromCOVID-19 ( $p=0.025)$ faces than for COVID-19-free faces. The interaction effect is shown in Figure 5(B). No main effect of "Immunological Status" was found.

\subsection{Post-experimental interview}


311 PEI responses are summarised in Table 3. A principal component analysis was

312 conducted to assess the dimensional structure of the six risk-perception items.

313 Preliminarily, we checked data for sampling adequacy and sphericity: the KMO value

314 was .59 and Bartlett's test of sphericity was significant $(X 2(15)=44.32, p=0.001)$, thus

315 indicating that the sampling was adequate and the inter-item correlations were large

316 enough, hence suggesting that a principal component analysis was appropriate. The

317 number of factors retained was determined through parallel analysis (Dinno, 2009;

318 Horn, 1965), inspection of scree plots and interpretability of each component. The

319 parallel analysis suggested a two-factor solution accounting for $58 \%$ of the total

320 variance, but both the scree plot and the components' interpretability supported instead

321 the extraction of three factors, which accounted for $73 \%$ of the total variance (Table 4).

322 Items 3.1, 3.4, and 3.6 represented the Severity dimension (Cronbach's $\alpha=.68$ ), items

3233.2 and 3.3 represented the Probability dimension (Cronbach's $\alpha=.45$ ), and item 3.5

324 the Knowledge dimension. Scores of section 4 and 5 items were aggregated separately

325 to create a COVID-19-related behaviour scale (Cronbach's $\alpha=.60$ ) and a COVID-19-

326 related intention scale (Cronbach's $\alpha=.71$ ), respectively. Pearson's correlation

327 coefficients among the PEI scales and the gaze-behavioural data are reported in Table

328 5. An interesting pattern emerged, with face fixation duration and scores in the

329 perceived Severity being negatively correlated with fixations to Eye AOls and positively

330 correlated with fixations to Mouth AOIs (both at $500 \mathrm{~ms}$ and $5000 \mathrm{~ms}$ ), although the only

331 statistically significant correlations were with the Mouth $\mathrm{AOI}$ in the recovered-from-

332 COVID-19 face condition (at $5000 \mathrm{~ms}, r=.41, p=0.003$ ) and with the Eye AOI in the

333 COVID-19-free face condition (at $500 \mathrm{~ms}, r=-.29, p=0.043$ ). No significant correlations

334 were found with the other dimensions of risk perception, nor with the COVID-19-related

335 behaviour and intention scales.

\section{4. Discussion}

337 In this study, we examined how participants looked at faces of individuals who could be 338 presented as "COVID-19 Free", "Sick with COVID-19", or "Recovered from COVID-19". 339 In so doing, we aimed at investigating how the multitude of psychosocial effects 340 produced by the current pandemic (Bagcchi, 2020; Bavel et al., 2020) and the related 
341 state of generalised apprehension and fear (Pakpour \& Griffiths, 2020) may reverberate 342 on daily-life, socially-based human cognitive functioning. Specifically, we chose to 343 analyse how the current dramatic contingencies may affect the way people interact with 344 one another. We focused our attention on how humans look at others' faces because 345 eye-contact effects constitute one of the most powerful and human-characterising social 346 behaviours (Csibra \& Gergely, 2006; Hernandez et al., 2009; Kleinke, 1986; Mertens et al., 1993; Senju \& Johnson, 2009a; Walker-Smith et al., 1977; Wirth et al., 2010; Conty et al., 2016; Dalmaso et al., 2020). Therefore, we posited that faces of people who were considered possible threatening stimuli due to their COVID-19-related illness might become the target of distinctive visual-attention patterns as compared to the more

351

352

353

354

355

356

357

358

359

360

361

362

363

364

365

366

367

368

369

370

371 reassuring faces of COVID-19-free individuals, possibly because COVID-19-related faces activate implicit cognitive mechanisms associated with risk avoidance and fear (Adolphs, 2009, 2010; Hietanen, 2018; Johnson et al., 2005; Kawashima et al., 1999; LeDoux, 2003; Lin et al., 2009; Loftus et al., 1987; Misslin, 2003; Skuse, 2003).

Consistent with our predictions, participants looked at the eyes of faces presented as "COVID-19 Free" significantly longer than faces presented as "Sick with COVID-19" or "Recovered from COVID-19". Such a peculiar visual-attentional pattern seems to emerge from the very first visual exploration time interval (i.e., 500ms), suggesting, as an at-first-glance indication, an initial detachment-from-threateningstimuli mechanism (see Figure 5A). However, and most important, by extending the time window of the eye-tracking analysis to $5000 \mathrm{~ms}$ - thus covering participants' full visual exploration - we found the same higher eye-focused pattern for COVID-19-free faces. Notably, within the extended time window, the main effect of AO/s with eyes receiving longer fixations than mouth was mitigated by a significant interaction. Thus, when considering both time windows of analysis, results clearly indicate that participants' implicit visual-attention patterns were modulated by explicit information on the health status of to-look-at faces, with a higher amount of eye contact for COVID-19free faces than for both sick-with/recovered-from-COVID-19 faces.

Along with differences in eye contact as a function of health status, within the extended time window of analysis (5000ms), we found a longer time allocation of visualspatial attention for the mouth's face area under both sick-with-COVID-19 and 
372 recovered-from-COVID-19 conditions as compared to the COVID-19-free condition.

373 Interestingly, participants visually explored the eyes and the mouth of COVID-19-related

374 faces in a complementary way. Indeed, participants looked at the eyes of COVID-19-

375 related faces about 200ms less than COVID-19-free faces. Symmetrically, participants

376 fixated the mouth of COVID-19-related faces about 200ms longer than COVID-19-free

377 ones (see Figure 5B). Thus, besides the implicit and sudden detachment-from-eyes

378 mechanism, shorter eye fixation strongly supports the idea of a greater attraction

379 exerted by the mouth in the quality of the threat-related area of the stimulus (Lin et al.,

380 2009; Loftus et al., 1987). Indeed, as we stated above, SARS-CoV-2 may spread

381 through respiratory droplets and aerosols (World Health Organization, 2020b). Such

382 contagion-related information is nowadays part of the semantic reservoir of the general

383 population. Therefore, one may reasonably assume how the threatening

384 characterisation of the mouth may depend on the specific transmission modality of the

385 virus (i.e., airborne). Whereas this study represents the first exploration of such a

386 complex phenomenon in the context of the COVID-19 pandemic, future studies should

387 explicitly explore the risk-perception mechanisms associated with other diseases.

Critically, we found overlapping results for sick-with-COVID-19 and recovered-

389 from-COVID-19 conditions for all the effects we found. Whereas such overlap might affect the stigma associated with COVID-19 (Bagcchi, 2020), it may also depend on the compromised and uncertainty-governed informative context generated by the pandemic (Koffman et al., 2020). Indeed, the massive profusion of often inaccurate, or even fake, news about the disease outcomes and the transmission modalities may have led people to take an incorrect, anxiety-modulated precautionary attitude towards the individuals recovered from COVID-19 (Usher et al., 2020; van der Linden et al., 2020). In this regard, it should be noticed that individuals largely use Internet and social media to obtain information regarding COVID-19. The ability to check for the correctness of Internet-distributed information is inevitably limited so that the risk to get fake news is consistently high (Lazer et al., 2018). Also, the large availability of COVID-19-related information may generate information overload and overconcern among people, thus fuelling the fear of COVID-19 (Farooq et al., 2020). 
Individuals are frightened by COVID-19 (Pakpour \& Griffiths, 2020). To date, the

403

404

405

406

407

408

409

410

411

412

413

414

415

416

417

418

419

420

421

422

423

424

425

426

427

428

429

430

431

432

fear of COVID-19 has led some individuals to commit suicide (Goyal et al., 2020; Mamun \& Griffiths, 2020) and the size of the problem is so large that some scholars have built ad-hoc scales (Ahorsu et al., 2020). Based on that knowledge, besides gazebehavioural measures, we included some ad-hoc psychometric measures to explore the perceived risk of COVID-19 contagion. The factorial analysis of participants' postexperimental-interview (PEI) revealed three risk-related main factors (i.e., perceived severity of COVID-19 contagion, probability of controlling the risk of infection, and knowledge about COVID-19) that explain over $73 \%$ of variance. This result is consistent with the typical factorial structures reported in the risk-perception literature, which include both cognitive (knowledge and probability of controlling the risk) and emotional (severity) dimensions (Loewenstein et al., 2001; Oh et al., 2015; Slovic, 1987, 2016).

The idea that fear might be at the root of the overlapping results for sick-with-COVID-19 and recovered from COVID-19 seems to be further supported by the correlation $(r=.41)$ between perceived severity and time of observation of the Mouth AOls of recoveredfrom-COVID-19 faces. Also, within our sample, the perceived severity of COVID-19 contagion was negatively correlated with fixations to the eyes and positively correlated with fixations to the mouth, within both time windows of analysis. However, it should be noted that, although adequate for studying eye movements, this study's sample size is too small to measure individual psychosocial differences. Therefore, the correlation analyses we propose here should be considered only as a non-exhaustive support to interpret and discuss the gaze-behavioural effects we found, which constitute the study's main result. Thus, by underlining how the most critical findings of the present research are those related to how participants changed their visual-attentional patterns as a function of faces' COVID-19 immunological status, the PEI data should be considered as potentially fruitful direction for future research aimed at exploring the most profound psychosocial implications of COVID-19.

To sum up, the interaction we found between immunological status and how participants looked at different characteristics of the faces suggests that the COVID-19related contingencies we are experiencing may resonate on basic cognitive processing underlying social interaction. Indeed, in our study, participants' performance in an 
433 implicit task (free face observation) was substantially influenced by explicit information 434 (immunological status) provided prior to the test. Persuaded by the evidence 435 accumulated during the last twenty years of neuroscientific research, we are keen to 436 interpret our results in terms of abrupt triggering of the neurocognitive systems involved 437 in social functioning, fear and gaze-behavioural control (Adolphs, 2009, 2010; Hietanen, 438 2018; Johnson et al., 2005; LeDoux, 2003; Lin et al., 2009; Loftus et al., 1987; Misslin, 439 2003; Senju \& Johnson, 2009a; Skuse, 2003; Wirth et al., 2010). By providing the first 440 evidence about the effects of the pandemic on the most basic level of social cognition 441 (i.e., eye contact), the present research shed new light on how flexible and adaptive 442 cognitive processing may lead humans to interact with the environment in a plastic way, 443 by integrating multiple sources of information (Federico et al., 2021; Federico \& 444 Brandimonte, 2020).

\section{Conclusions}

COVID-19 pandemic produced and is still producing strong psychosocial effects within the general population. Whereas current research has addressed the clinical and biopsycho-sociological effects of COVID-19, much less space has been devoted to the pandemic's consequences on non-pathological and daily-life cognitive functions. In the context of the neuroscience/psychological perspective of social cognition, we investigated whether and, if so, how humans modify their social-interaction modalities due to the COVID-19-related contingencies. In particular, we analysed how people looked at faces of individuals presented as COVID-19 free, sick with COVID-19, or recovered from COVID-19. We found that participants tended to look at the eyes of COVID-19-free faces longer than at those of both COVID-19-related faces. Crucially, under both COVID-19-related conditions, the implicit detachment-from-eyes mechanism we report seems to be compensated by increasing visual attention to the mouth area. This increase suggests a threatening characterisation of the mouth as a transmission vehicle for SARS-CoV-2. Notably, such an implicit gaze-behavioural pattern appears to be consistent with the self-report psychometric measures we introduced to find out how participants perceived the risk of COVID-19 contagion. As an initial exploration of a very complex reality, this article reports the first evidence in the literature about the 
463 pandemic's psychological and social reverberations on the most basic level of human 464 social interaction. 
466

467

468

469

470

471

472

473

474

475

476

477

478

479

480

481

482

483

484

485

486

487

488

489

490

491

492

493

494

\section{References}

- Adolphs, R. (2009). The Social Brain: Neural Basis of Social Knowledge. Annual Review of Psychology, 60(1). https://doi.org/10.1146/annurev.psych.60.110707.163514

- Adolphs, R. (2010). What does the amygdala contribute to social cognition? Annals of the New York Academy of Sciences, 1191(1). https://doi.org/10.1111/j.1749$6632.2010 .05445 . x$

- Ahorsu, D. K., Lin, C.-Y., Imani, V., Saffari, M., Griffiths, M. D., \& Pakpour, A. H. (2020). The Fear of COVID-19 Scale: Development and Initial Validation. International Journal of Mental Health and Addiction. https://doi.org/10.1007/s11469-020-00270-8

- American Psychiatric Association. (2013). Diagnostic and Statistical Manual of Mental Disorders. American Psychiatric Association. https://doi.org/10.1176/appi.books.9780890425596

- Auyeung, B., Lombardo, M. v, Heinrichs, M., Chakrabarti, B., Sule, A., Deakin, J. B., Bethlehem, R. A. I., Dickens, L., Mooney, N., Sipple, J. A. N., Thiemann, P., \& Baron-Cohen, S. (2015). Oxytocin increases eye contact during a real-time, naturalistic social interaction in males with and without autism. Translational Psychiatry, 5(2). https://doi.org/10.1038/tp.2014.146

- Bagcchi, S. (2020). Stigma during the COVID-19 pandemic. The Lancet Infectious Diseases, 20(7). https://doi.org/10.1016/S1473-3099(20)30498-9

- Bavel, J. J. van, Baicker, K., Boggio, P. S., Capraro, V., Cichocka, A., Cikara, M., Crockett, M. J., Crum, A. J., Douglas, K. M., Druckman, J. N., Drury, J., Dube, O., Ellemers, N., Finkel, E. J., Fowler, J. H., Gelfand, M., Han, S., Haslam, S. A., Jetten, J., ... Willer, R. (2020). Using social and behavioural science to support COVID-19 pandemic response. Nature Human Behaviour, 4(5). https://doi.org/10.1038/s41562-020-0884-z

- Bhardwaj, R., \& Agrawal, A. (2020). Tailoring surface wettability to reduce chances of infection of COVID-19 by a respiratory droplet and to improve the effectiveness 
495 of personal protection equipment. Physics of Fluids, 32(8).

496 https://doi.org/10.1063/5.0020249

497 - Brooks, S. K., Webster, R. K., Smith, L. E., Woodland, L., Wessely, S., Greenberg,

498 N., \& Rubin, G. J. (2020). The psychological impact of quarantine and how to

499 reduce it: rapid review of the evidence. The Lancet, 395(10227).

500 https://doi.org/10.1016/S0140-6736(20)30460-8

501 - Burra, N., Hervais-Adelman, A., Kerzel, D., Tamietto, M., de Gelder, B., \& Pegna,

502 A. J. (2013). Amygdala Activation for Eye Contact Despite Complete Cortical

503 Blindness. Journal of Neuroscience, 33(25).

$504 \quad$ https://doi.org/10.1523/JNEUROSCI.3994-12.2013

505 - Cohen, J. (2013). Statistical Power Analysis for the Behavioral Sciences.

$506 \quad$ Routledge. https://doi.org/10.4324/9780203771587

507 - Cousineau, D. (2005). Confidence intervals in within-subject designs: A simpler

508 solution to Loftus and Masson's method. Tutorials in quantitative methods for

509 psychology, 1(1), 42-45.

510 - Csibra, G., \& Gergely, G. (2006). Social learning and social cognition: The case for

511 pedagogy. In

512 - Conty, L., George, N., \& Hietanen, J. K. (2016). Watching Eyes effects: When

513 others meet the self. Consciousness and cognition, 45, 184-197.

514 - Dalmaso, M., Castelli, L., \& Galfano, G. (2020). Social modulators of gaze-

515 mediated orienting of attention: A review. Psychonomic bulletin \& review, 27, 833-

516855.

517 - Dinno, A. (2009). Exploring the sensitivity of Horn's parallel analysis to the

518 distributional form of random data. Multivariate behavioral research, 44(3), 362-

519388.

520 - Emery, N. J. (2000). The eyes have it: the neuroethology, function and evolution of

521 social gaze. Neuroscience \& Biobehavioral Reviews, 24(6).

522 https://doi.org/10.1016/S0149-7634(00)00025-7

523 - Farooq, A., Laato, S., \& Islam, A. K. M. N. (2020). Impact of Online Information on

524 Self-Isolation Intention During the COVID-19 Pandemic: Cross-Sectional Study.

525 Journal of Medical Internet Research, 22(5). https://doi.org/10.2196/19128 
526 - Faul, F., Erdfelder, E., Lang, A.-G., \& Buchner, A. (2007). G*Power 3: A flexible

527 statistical power analysis program for the social, behavioral, and biomedical

528 sciences. Behavior Research Methods, 39(2). https://doi.org/10.3758/BF03193146

529 - Federico, G., \& Brandimonte, M. A. (2019). Tool and object affordances: An

530 ecological eye-tracking study. Brain and Cognition, 135.

531 https://doi.org/10.1016/j.bandc.2019.103582

532 - Federico, G., \& Brandimonte, M. A. (2020). Looking to recognise: the pre-eminence 533 of semantic over sensorimotor processing in human tool use. Scientific Reports,

534 10(1). https://doi.org/10.1038/s41598-020-63045-0

535 - Federico, G., Osiurak, F., \& Brandimonte, M. A. (2021). Hazardous tools: the

536 emergence of reasoning in human tool use. Psychological Research.

537 https://doi.org/10.1007/s00426-020-01466-2

538 - Fiorillo, A., \& Gorwood, P. (2020). The consequences of the COVID-19 pandemic

539 on mental health and implications for clinical practice. European Psychiatry, 63(1).

$540 \quad$ https://doi.org/10.1192/j.eurpsy.2020.35

541 - Goyal, K., Chauhan, P., Chhikara, K., Gupta, P., \& Singh, M. P. (2020). Fear of

542 COVID 2019: First suicidal case in India! Asian Journal of Psychiatry, 49.

$543 \quad$ https://doi.org/10.1016/j.ajp.2020.101989

544 - Hayhoe, M., \& Ballard, D. (2005). Eye movements in natural behavior. Trends in

545 Cognitive Sciences, 9(4). https://doi.org/10.1016/j.tics.2005.02.009

546 - Henderson, J. M. (2017). Gaze Control as Prediction. Trends in Cognitive

$547 \quad$ Sciences, 21(1). https://doi.org/10.1016/j.tics.2016.11.003

548 - Hernandez, N., Metzger, A., Magné, R., Bonnet-Brilhault, F., Roux, S., Barthelemy, 549 C., \& Martineau, J. (2009). Exploration of core features of a human face by healthy 550 and autistic adults analyzed by visual scanning. Neuropsychologia, 47(4).

551 https://doi.org/10.1016/j.neuropsychologia.2008.10.023

552 - Hietanen, J. K. (2018). Affective Eye Contact: An Integrative Review. Frontiers in 553 Psychology, 9. https://doi.org/10.3389/fpsyg.2018.01587

554 - Horn, J. L. (1965). A rationale and test for the number of factors in factor analysis. 555 Psychometrica, 30(2), 179-185. 
556 - Insel, T. R. (2010). The Challenge of Translation in Social Neuroscience: A Review

557 of Oxytocin, Vasopressin, and Affiliative Behavior. Neuron, 65(6).

$558 \quad$ https://doi.org/10.1016/j.neuron.2010.03.005

559 - Johnson, M. H., Griffin, R., Csibra, G., Halit, H., Farroni, T., De Haan, M., Tucker, 560 L. A., Baron-Cohen, S., \& Richards, J. (2005). The emergence of the social brain 561 network: Evidence from typical and atypical development. Development and $562 \quad$ Psychopathology, 17(03). https://doi.org/10.1017/S0954579405050297

- Just, M. A., \& Carpenter, P. A. (1976). The role of eye-fixation research in cognitive 564 psychology. Behavior Research Methods \& Instrumentation, 8(2). https://doi.org/10.3758/BF03201761

- Karras, T., Laine, S., Aittala, M., Hellsten, J., Lehtinen, J., \& Aila, T. (2020, June). Analyzing and Improving the Image Quality of StyleGAN. 2020 IEEE/CVF Conference on Computer Vision and Pattern Recognition (CVPR).

- Kawashima, R., Sugiura, M., Kato, T., Nakamura, A., Hatano, K., Ito, K., Fukuda, H., Kojima, S., \& Nakamura, K. (1999). The human amygdala plays an important role in gaze monitoring. Brain, 122(4). https://doi.org/10.1093/brain/122.4.779

- Kleinke, C. L. (1986). Gaze and eye contact: A research review. Psychological Bulletin, 100(1). https://doi.org/10.1037/0033-2909.100.1.78

- Koffman, J., Gross, J., Etkind, S. N., \& Selman, L. (2020). Uncertainty and COVID19: how are we to respond? Journal of the Royal Society of Medicine, 113(6). https://doi.org/10.1177/0141076820930665

- Lazer, D. M. J., Baum, M. A., Benkler, Y., Berinsky, A. J., Greenhill, K. M., Menczer, F., Metzger, M. J., Nyhan, B., Pennycook, G., Rothschild, D., Schudson, M., Sloman, S. A., Sunstein, C. R., Thorson, E. A., Watts, D. J., \& Zittrain, J. L. (2018). The science of fake news. Science, 359(6380). https://doi.org/10.1126/science.aao2998

- LeDoux, J. (2003). The emotional brain, fear, and the amygdala. Cellular and Molecular Neurobiology, 23(4/5). https://doi.org/10.1023/A:1025048802629

- Lin, J. Y., Murray, S. O., \& Boynton, G. M. (2009). Capture of Attention to Threatening Stimuli without Perceptual Awareness. Current Biology, 19(13). 
587 - Liversedge, S. P., \& Findlay, J. M. (2000). Saccadic eye movements and cognition.

588 Trends in Cognitive Sciences, 4(1). https://doi.org/10.1016/S1364-6613(99)01418-7

589 - Loftus, G. R., \& Masson, M. E. (1994). Using confidence intervals in within-subject 590 designs. Psychonomic bulletin \& review, 1(4), 476-490.

591 - Loewenstein, G. F., Weber, E. U., Hsee, C. K., \& Welch, N. (2001). Risk as

592 feelings. Psychological Bulletin, 127(2). https://doi.org/10.1037/0033-

$593 \quad 2909.127 .2 .267$

594 - Loftus, E. F., Loftus, G. R., \& Messo, J. (1987). Some facts about "weapon focus."

$595 \quad L a w$ and Human Behavior, 11(1). https://doi.org/10.1007/BF01044839

596 - Mamun, M. A., \& Griffiths, M. D. (2020). First COVID-19 suicide case in

597 Bangladesh due to fear of COVID-19 and xenophobia: Possible suicide prevention

$598 \quad$ strategies. Asian Journal of Psychiatry, 51.

$599 \quad$ https://doi.org/10.1016/j.ajp.2020.102073

600 - Mertens, I., Siegmund, H., \& Grüsser, O.-J. (1993). Gaze motor asymmetries in the 601 perception of faces during a memory task. Neuropsychologia, 31(9).

602 https://doi.org/10.1016/0028-3932(93)90154-R

603 - Milner, D., \& Goodale, M. (2006). The Visual Brain in Action. Oxford University 604 Press. https://doi.org/10.1093/acprof:oso/9780198524724.001.0001

605 - Misslin, R. (2003). The defense system of fear: behavior and neurocircuitry.

$606 \quad$ Neurophysiologie Clinique/Clinical Neurophysiology, 33(2).

607 https://doi.org/10.1016/S0987-7053(03)00009-1

608 - Oh, S.-H., Paek, H.-J., \& Hove, T. (2015). Cognitive and emotional dimensions of 609 perceived risk characteristics, genre-specific media effects, and risk perceptions:

610 the case of H1N1 influenza in South Korea. Asian Journal of Communication, 611 25(1). https://doi.org/10.1080/01292986.2014.989240

612 - Pakpour, A. H., \& Griffiths, M. D. (2020). The fear of COVID-19 and its role in 613 preventive behaviors. In Journal of Concurrent Disorders (Vol. 2, Issue 1).

614 - Papoutsaki, A., Daskalova, N., Sangkloy, P., Huang, J., Laskey, J., \& Hays, J. 615 (2016). WebGazer: Scalable Webcam Eye Tracking Using User Interactions. 616 Proceedings of the Twenty-Fifth International Joint Conference on Artificial 617 Intelligence-IJCAI 2016. 
618 - Pfefferbaum, B., \& North, C. S. (2020). Mental Health and the Covid-19 Pandemic.

619 New England Journal of Medicine, 383(6). https://doi.org/10.1056/NEJMp2008017

620 - Rahal, R.-M., \& Fiedler, S. (2019). Understanding cognitive and affective

621 mechanisms in social psychology through eye-tracking. Journal of Experimental

622 Social Psychology, 85. https://doi.org/10.1016/j.jesp.2019.103842

623 - Rayner, K. (1998). Eye movements in reading and information processing: 20 years

624 of research. Psychological Bulletin, 124(3). https://doi.org/10.1037/0033-

$625 \quad 2909.124 .3 .372$

626 - Semmelmann, K., \& Weigelt, S. (2018). Online webcam-based eye tracking in

627 cognitive science: A first look. Behavior Research Methods, 50(2), 451-465.

628 - Senju, A., \& Johnson, M. H. (2009a). The eye contact effect: mechanisms and

629 development. Trends in Cognitive Sciences, 13(3).

630 https://doi.org/10.1016/j.tics.2008.11.009

631 - Senju, A., \& Johnson, M. H. (2009b). Atypical eye contact in autism: Models, 632 mechanisms and development. Neuroscience \& Biobehavioral Reviews, 33(8).

633 https://doi.org/10.1016/j.neubiorev.2009.06.001

634 - Senju, A., Johnson, M. H., \& Csibra, G. (2006). The development and neural basis

635 of referential gaze perception. Social Neuroscience, 1(3-4).

636 https://doi.org/10.1080/17470910600989797

637 - Skuse, D. (2003). Fear Recognition and the Neural Basis of Social Cognition. Child 638 and Adolescent Mental Health, 8(2). https://doi.org/10.1111/1475-3588.00047

639 - Slovic, P. (1987). Perception of risk. Science, 236(4799).

640 https://doi.org/10.1126/science.3563507

641 - Slovic, P. (2016). The Perception of Risk. Routledge.

642 https://doi.org/10.4324/9781315661773

643 - Usher, K., Jackson, D., Durkin, J., Gyamfi, N., \& Bhullar, N. (2020).

644 Pandemic-related behaviours and psychological outcomes; A rapid literature review 645 to explain COVID-19 behaviours. International Journal of Mental Health Nursing, 646 29(6). https://doi.org/10.1111/inm.12790 
647 - van der Linden, S., Roozenbeek, J., \& Compton, J. (2020). Inoculating Against

$648 \quad$ Fake News About COVID-19. Frontiers in Psychology, 11.

649 https://doi.org/10.3389/fpsyg.2020.566790

650 - Walker-Smith, G. J., Gale, A. G., \& Findlay, J. M. (1977). Eye Movement Strategies

651 Involved in Face Perception. Perception, 6(3). https://doi.org/10.1068/p060313

652 - Wirth, J. H., Sacco, D. F., Hugenberg, K., \& Williams, K. D. (2010). Eye Gaze as

653 Relational Evaluation: Averted Eye Gaze Leads to Feelings of Ostracism and

654 Relational Devaluation. Personality and Social Psychology Bulletin, 36(7).

655 https://doi.org/10.1177/0146167210370032

656 - World Health Organization. (2020a). Coronavirus disease (COVID-19).

657 https://www.who.int/emergencies/diseases/novel-coronavirus-2019/question-and-

658 answers-hub/q-a-detail/coronavirus-disease-covid-19

659 - World Health Organization. (2020b). Q\&A: How is COVID-19 transmitted?

660 https://www.who.int/vietnam/news/detail/14-07-2020-q-a-how-is-covid-19-

661 transmitted

662

663 


\section{Figure 1}

Example of stimuli used in the study

Example of face stimuli used in the study $(A-F)$. All the faces were generated through machine learning by implementing a generative adversarial network (Karras et al., 2020).

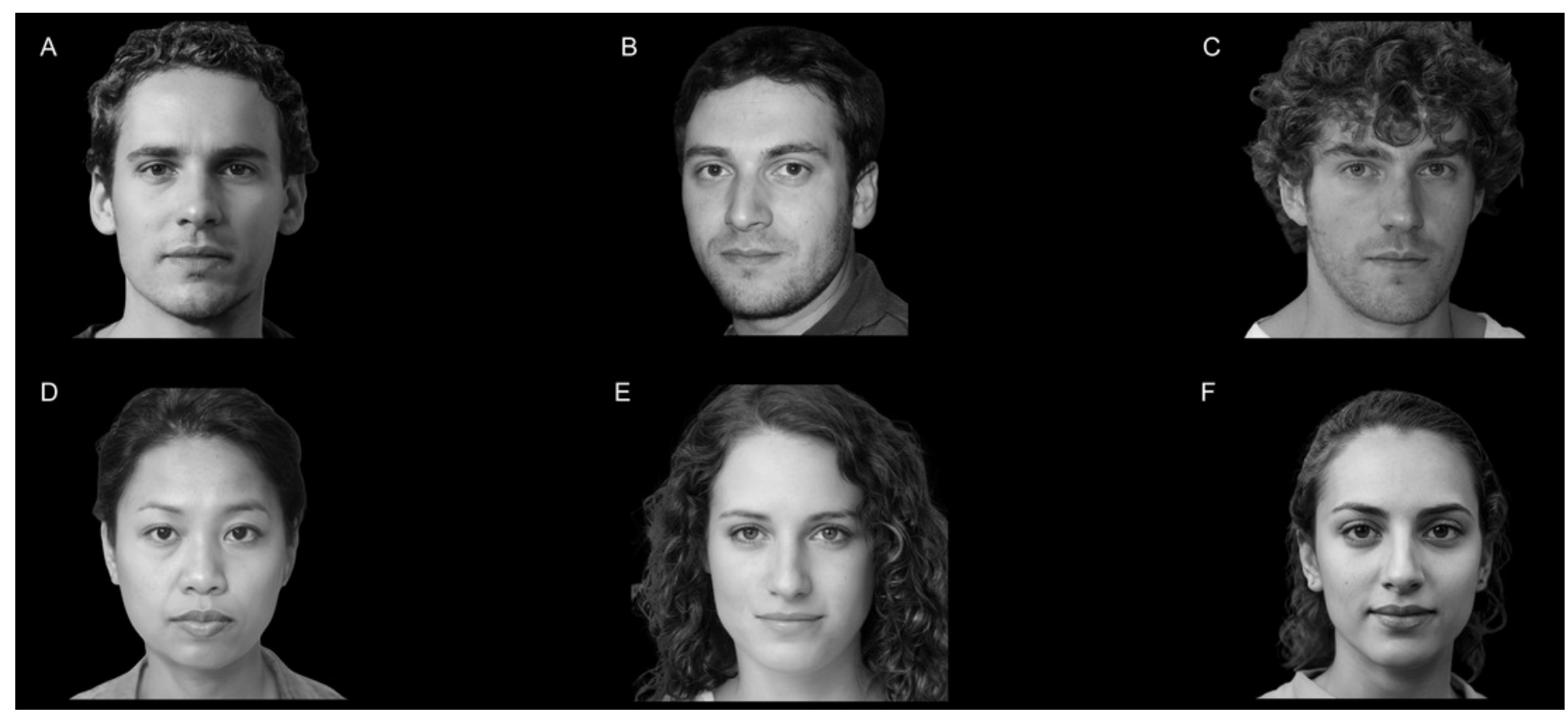




\section{Figure 2}

\section{Experimental flow}

A fixation point appeared for $500 \mathrm{~ms}$, followed by a label indicating the Immunological Status (i.e., "COVID-19 free", "Sick with COVID-19", or "Recovered from COVID-19"), which was shown for $2000 \mathrm{~ms}$. Then, a second fixation point $(500 \mathrm{~ms})$ appeared followed by a face stimulus that remained on the screen for $5000 \mathrm{~ms}$. Finally, a black screen appeared for $4000 \mathrm{~ms}$.

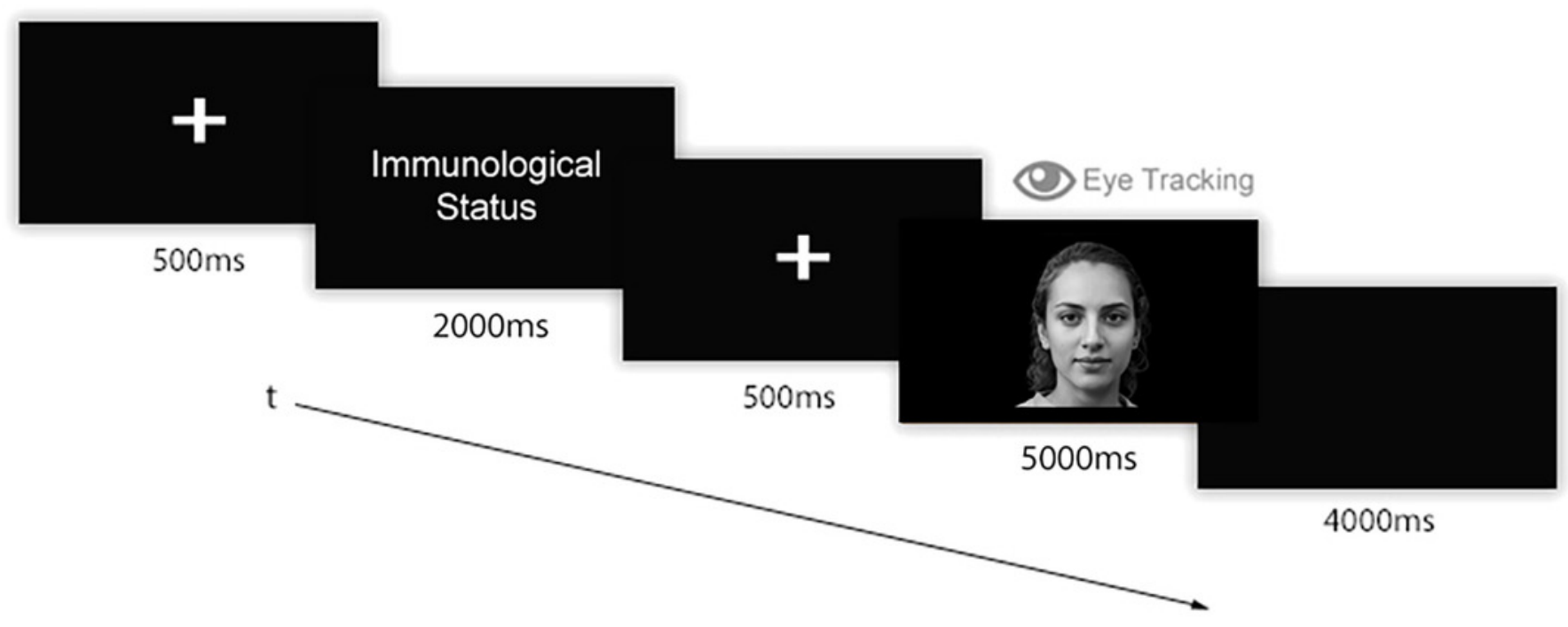




\section{Figure 3}

Face AOls considered in the study

We analysed how participants looked at two distinct AOls of the faces involved in the study. The first AOI was associated with the eyes (i.e., "Eye AOI"). The second AOI referred to the mouth (i.e., "Mouth AOI"). Both AOIs remained stable in terms of size, spatial position, and proportions across the stimuli.

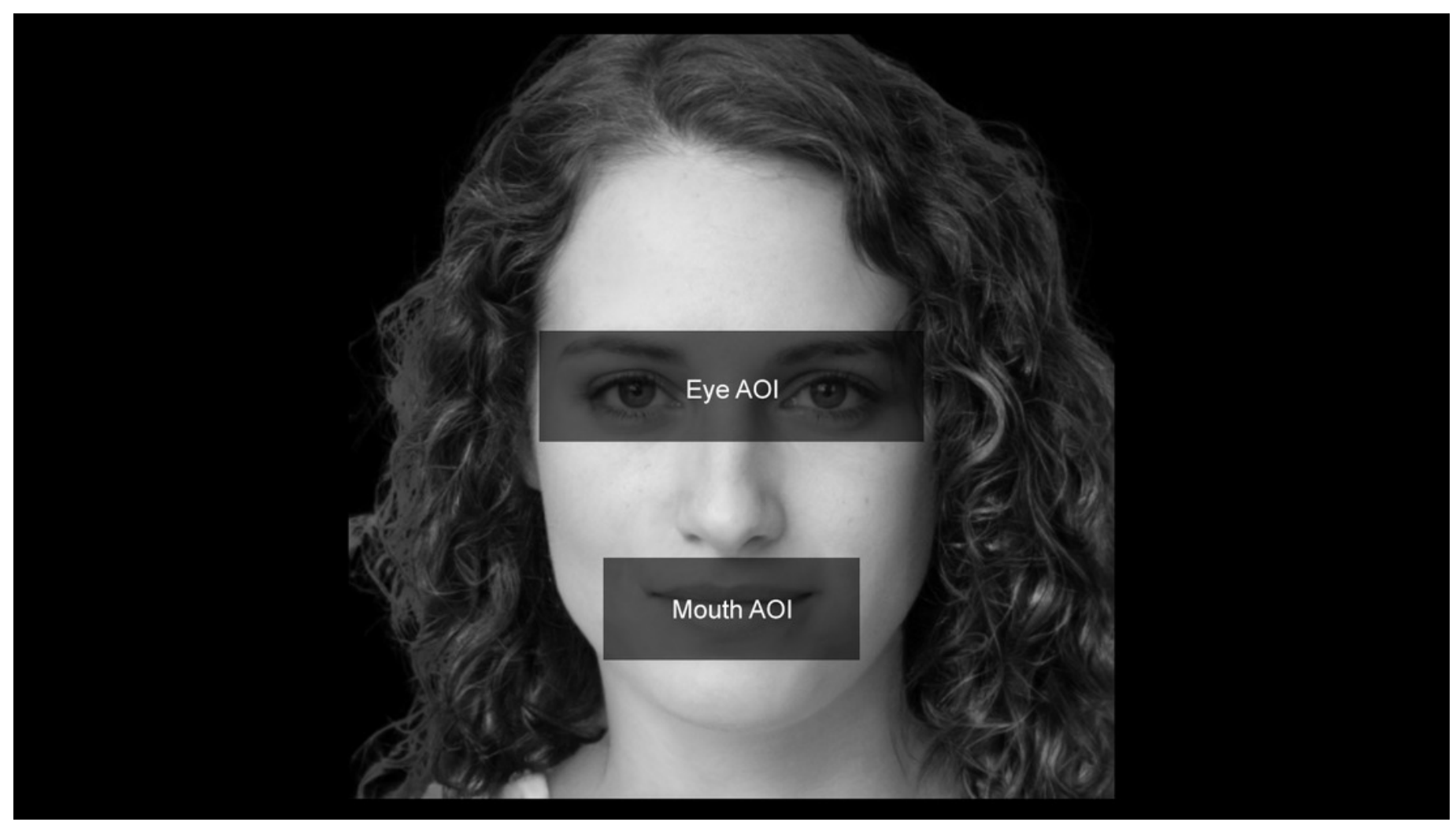




\section{Figure 4}

Face exploration heatmaps

Example of heatmaps related to how all participants explored faces of individuals that could be presented as (A) COVID-19 free, (B) Sick with COVID-19, or (C) Recovered from COVID-19. Time window: 5000ms.

(A)

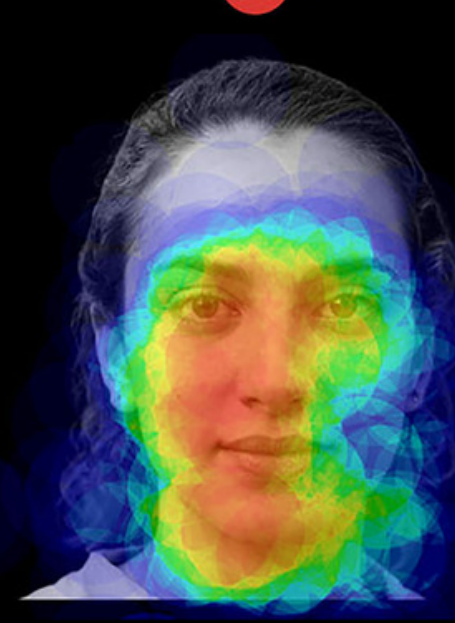

B

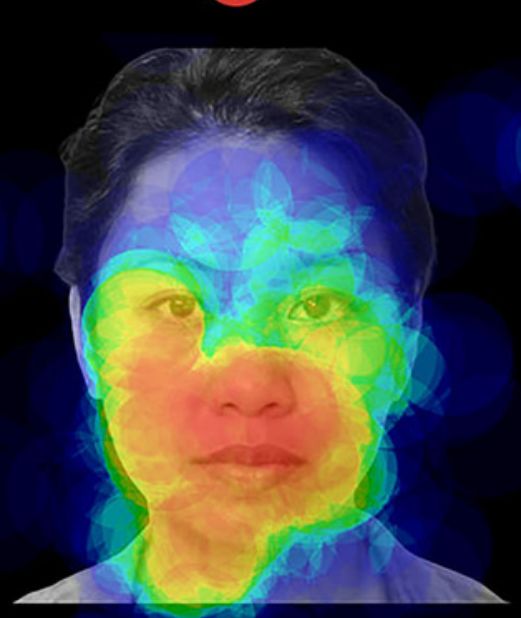

(C)

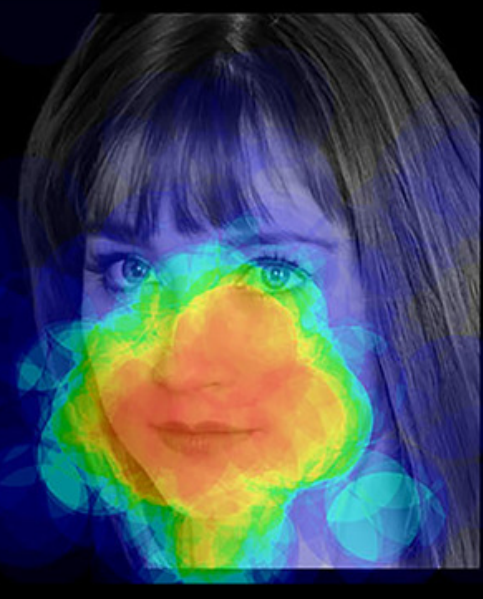




\section{Figure 5}

The effect of COVID-19 Immunological Status on face visual exploration

Participants looked at the eyes of individuals presented as "COVID-19 Free" longer than at those of individuals presented as "Sick with COVID-19" or "Recovered from COVID-19" for both time windows of analysis ( $A$ and $B$ ). Additionally, the full exploration analysis (B) revealed that mouth areas of both sick-wih-COVID-19 and recovered-from-COVID-19 faces were fixated longer than COVID-19-free ones. Vertical bars denote .95 confidence intervals, computed by adopting a simpler solution to Loftus \& Masson (1994) provided by Cousineau (2005).

A

Initial visual exploration $(\mathrm{t}=500 \mathrm{~ms})$

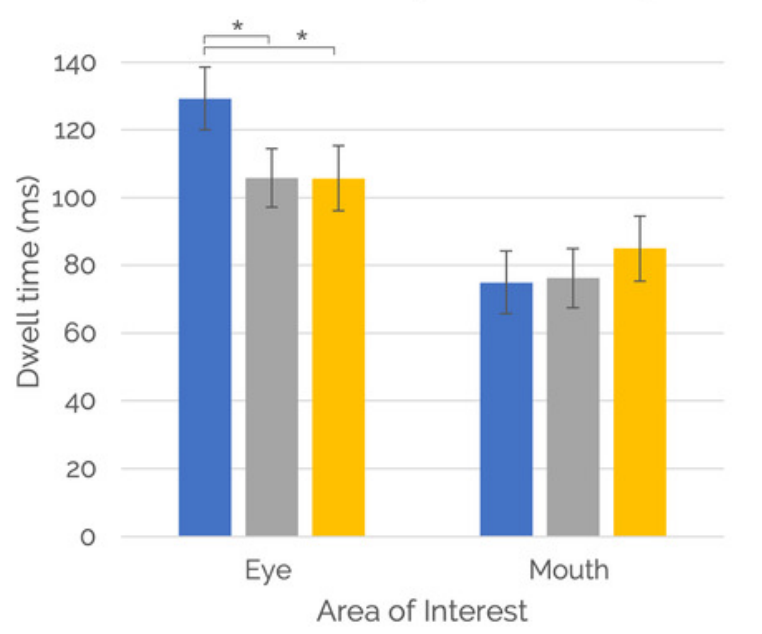

B

Full visual exploration $(\mathrm{t}=5000 \mathrm{~ms})$

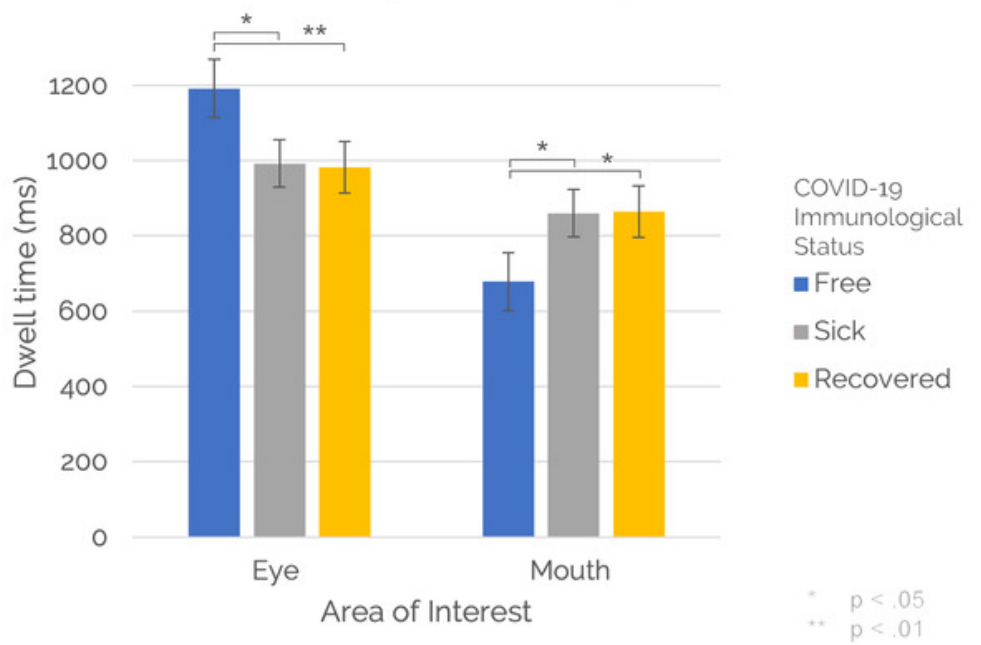


Table 1 (on next page)

Initial visual exploration (500ms)

Eye-tracking data (sampling rate: $20 \mathrm{~Hz}$ ) related to participants' initial visual exploration. 


\begin{tabular}{|r|r|r|}
\hline & \multicolumn{2}{|c|}{ AOls - Dwell Time (SD) } \\
\hline Immunological Status & Eyes & Mouth \\
\hline COVID-19 FREE & $129,26(86,06) \mathrm{ms}$ & $74,97(59,44) \mathrm{ms}$ \\
\hline SICK WITH COVID-19 & $105,86(80,4) \mathrm{ms}$ & $76,23(56,11) \mathrm{ms}$ \\
\hline RECOVERED FROM COVID-19 & $105,71(83,36) \mathrm{ms}$ & $84,96(68,19) \mathrm{ms}$ \\
\hline
\end{tabular}

1 


\section{Table 2 (on next page)}

Full visual exploration (5000ms)

Eye-tracking data (sampling rate: $20 \mathrm{~Hz}$ ) related to participants' full visual exploration. 


\begin{tabular}{|r|r|r|}
\hline & \multicolumn{3}{|r|}{ AOls - Dwell Time (SD) } \\
\hline Immunological Status & Eyes & Mouth \\
\hline COVID-19 FREE & $1191,48(790,63) \mathrm{ms}$ & $678,25(403,02) \mathrm{ms}$ \\
\hline SICK WITH COVID-19 & $991,99(670,22) \mathrm{ms}$ & $859,96(391,528) \mathrm{ms}$ \\
\hline RECOVERED FROM COVID-19 & $982,39(710,69) \mathrm{ms}$ & $864,87(424,02) \mathrm{ms}$ \\
\hline
\end{tabular}

1 
Table 3 (on next page)

Post-experimental Interview results 
1

\begin{tabular}{|c|c|c|}
\hline Item & & \\
\hline COVID-19-related information & Yes (\%) & No $(\%)$ \\
\hline 2.1 I am currently COVID-19 positive & $1(2)$ & $48(98)$ \\
\hline 2.2 I got sick of COVID-19, but now I'm cured and negative & $3(6.1)$ & $\begin{array}{r}46 \\
(93.9) \\
\end{array}$ \\
\hline 2.3 In my circle of acquaintances, someone got sick of COVID-19 & $42(85.7)$ & $7(14.3)$ \\
\hline 2.4 In my circle of acquaintances, someone has died from COVID-19 & $14(28.6)$ & $\begin{array}{r}35 \\
(71.4)\end{array}$ \\
\hline Risk Perception & $\mathbf{M}$ & SD \\
\hline $\begin{array}{l}\text { 3.1 How risky is it for you coming into contact with a COVID-19 } \\
\text { patient? }\end{array}$ & 5.45 & 1.51 \\
\hline $\begin{array}{l}\text { 3.2 How likely is it for you coming into contact with a COVID-19 } \\
\text { patient? }\end{array}$ & 5.06 & 1.45 \\
\hline $\begin{array}{l}\text { 3.3 How much can you control the possibility of coming into contact } \\
\text { with a COVID-19 patient? }\end{array}$ & 4.27 & 1.30 \\
\hline 3.4 Are you afraid of coming into contact with a COVID-19 patient? & 4.82 & 1.72 \\
\hline $\begin{array}{l}\text { 3.5 Do you know what the consequences of coming into contact with a } \\
\text { COVID-19 patient are? }\end{array}$ & 6.33 & 0.92 \\
\hline $\begin{array}{l}\text { 3.6 How serious might be consequences you could have when coming } \\
\text { into contact with a COVID-19 patient? }\end{array}$ & 5.43 & 1.17 \\
\hline COVID-19-related behaviours & & \\
\hline 4.1 Used the mask & 5.86 & .35 \\
\hline 4.2 Used gloves & 2.06 & 1.25 \\
\hline 4.3 Used the hand sanitizer & 5.18 & 1.03 \\
\hline 4.4 Kept a distance of at least one meter from other people & 5.06 & .92 \\
\hline 4.5 Avoided crowded places & 5.33 & 0.80 \\
\hline 4.6 avoided meeting friends/relatives & 4.41 & 1.35 \\
\hline COVID-19-related intentions & & \\
\hline 5.1 Use the mask & 5.94 & 0.32 \\
\hline 5.2 Use gloves & 2.63 & 1.32 \\
\hline 5.3 Use the hand sanitizer & 5.47 & 0.77 \\
\hline 5.4 Keep a distance of at least one meter from other people & 5.33 & 0.80 \\
\hline 5.5 Avoid crowded places & 5.53 & 0.68 \\
\hline 5.6 Avoid meeting friends/relatives & 4.39 & 1.10 \\
\hline
\end{tabular}




\section{Table 4(on next page)}

Risk perception factor analysis

Risk perception factor analysis (extraction method: principal component analysis with varimax rotation). Component 1 has been named Severity, Component 2 Probability and Component 3 Knowledge. 


\begin{tabular}{|r|r|r|r|}
\hline & \multicolumn{3}{|c|}{ Components } \\
\hline Risk Perception Items & 1 & 2 & 3 \\
\hline 3.1 & .87 & .09 & -.04 \\
\hline 3.4 & .76 & -.18 & .17 \\
\hline 3.6 & .68 & .48 & .11 \\
\hline 3.3 & .13 & -.80 & .22 \\
\hline 3.2 & .15 & .76 & .12 \\
\hline 3.5 & .09 & -.03 & .98 \\
\hline
\end{tabular}

1 


\section{Table 5 (on next page)}

Pearson's correlation coefficients among the PEI scales and gaze-behavioural data

Notes: $*=p<.05, * *=p<.01$. 


\begin{tabular}{|c|c|c|c|c|c|}
\hline Item & Severity & Probability & Knowledge & $\begin{array}{r}\text { COVID-19- } \\
\text { related } \\
\text { behaviours }\end{array}$ & $\begin{array}{r}\text { COVID-19- } \\
\text { related } \\
\text { intentions }\end{array}$ \\
\hline Severity & - & & & & \\
\hline Probability & .13 & - & & & \\
\hline Knowledge & .19 & -.09 & - & & \\
\hline COVID-19-related behaviours & .17 & -.02 & .27 & - & \\
\hline COVID-19-related intentions & .23 & -.01 & .27 & $.84^{* *}$ & - \\
\hline $\begin{array}{r}\text { MFT COVID-19-FREE EYES } \\
500 \mathrm{~ms}\end{array}$ & $-.29 *$ & -.06 & -.03 & -.22 & -.13 \\
\hline $\begin{array}{r}\text { MFT COVID-19-FREE MOUTH } \\
500 \mathrm{~ms}\end{array}$ & .22 & .03 & .02 & -.06 & .00 \\
\hline $\begin{array}{r}\text { MFT COVID-19 SICK EYES } \\
500 \mathrm{~ms}\end{array}$ & -.19 & .03 & -.09 & -.14 & -.06 \\
\hline $\begin{array}{r}\text { MFT COVID-19 SICK MOUTH } \\
500 \mathrm{~ms}\end{array}$ & .15 & -.01 & -.05 & -.22 & -.08 \\
\hline $\begin{array}{r}\text { MFT COVID-19 RECOVERED } \\
\text { EYES 500ms }\end{array}$ & -.08 & .03 & .02 & -.03 & .07 \\
\hline $\begin{array}{r}\text { MFT COVID-19 RECOVERED } \\
\text { MOUTH 500ms }\end{array}$ & .18 & .01 & -.06 & -.23 & -.17 \\
\hline $\begin{array}{r}\text { MFT COVID-19-FREE EYES } \\
5000 \mathrm{~ms}\end{array}$ & -.17 & -.09 & -.01 & -.17 & -.07 \\
\hline $\begin{array}{r}\text { MFT COVID-19-FREE MOUTH } \\
5000 \mathrm{~ms}\end{array}$ & .18 & .11 & -13 & -.06 & .02 \\
\hline $\begin{array}{r}\text { MFT COVID-19 SICK EYES } \\
5000 m s\end{array}$ & -.14 & .03 & .07 & .01 & .11 \\
\hline $\begin{array}{r}\text { MFT COVID-19 SICK MOUTH } \\
5000 m s\end{array}$ & .04 & .04 & -.04 & -.11 & -.02 \\
\hline $\begin{array}{r}\text { MFT COVID-19 RECOVERED } \\
\text { EYES 5000ms }\end{array}$ & -.10 & -.08 & -.05 & -.02 & .07 \\
\hline $\begin{array}{r}\text { MFT COVID-19 RECOVERED } \\
\text { MOUTH 5000ms }\end{array}$ & $.41^{* *}$ & .07 & .08 & -.13 & -.10 \\
\hline
\end{tabular}

